\title{
Progressive Pseudorheumatoid Condrodysplasia, an Unusual Cause of Joint Swelling and Stiffness
}

\author{
(i) Esra Kılıç \\ University of Health Sciences, Ankara Child the alth and Diseases Hematology Oncology Training and Research Hospital, Clinic of Pediatric Genetics, \\ Ankara, Turkey
}

\begin{abstract}
Progressive pseudorheumatoid condrodysplasia (PPRC) is a rare autosomal recessive skeletal dysplasia characterized by joint swelling, stiffness, generalized progressive joint contractures and progressive arthropathy with the negative laboratory markers of juvenile idiopathic arthritis. It is caused by mutations of the Wnt1-inducible signalling pathway protein 3 (WISP3) gene located on chromosome 6q22, encoding a cysteine-rich connective tissue growth factor, which preserves cartilage integrity in chondrocytes. Here, we describe two new patients from same consanguineous family. The diagnosis was made by clinical and radiologic findings, molecular analysis of the WISP3 gene revealed a c.793-794 delTC (p.C265Lfs*31) mutation. Although life expectancy is normal, the prognosis of PPRC can be very poor, most patients are wheelchair-bound from an early age. Early recognition and appropriate genetic counselling is essential in order to avoid unnecessary treatment and further recurrence.

Keywords: Progressive pseudorheumatoid condrodysplasia, joint swelling, Wnt1-inducible signalling pathway protein 3
\end{abstract}

\section{Introduction}

Progressive pseudorheumatoid condrodysplasia (PPRC) (OMIM \#208230) is a rare progressive skeletal disorder due to autosomal recessive loss-of-function mutations of the Wnt1-inducible signalling pathway protein 3 (WISP3) gene on chromosome $6 \mathrm{q} 22(1,2)$. The WISP3 gene is a member of the connective tissue growth factor gene family and encodes a secreted cysteine-rich protein expressed by synoviocytes and chondrocytes (3). These proteins have roles in cell growth, differentiation and are essential for normal postnatal skeletal growth and cartilage homeostasis. The typical clinical manifestations and radiographic findings are progressive deformities, bone pain, stiffness and swelling of multiple joints with the absence of systemic or synovial inflammation, especially in the hips, knees, wrists and fingers, a limitation of motion, short stature, widened epiphyses, vertebral flattening, narrow joint spaces and osteoporosis
(4). Despite the lack of systemic and synovial inflammation, most of these patients are initially misdiagnosed as juvenile idiopathic arthritis (JIA). Here, we report on 2 siblings from a Turkish family diagnosed with PPRC.

\section{Case Report}

Patient 1 was a 7-year-old girl and patient 2 was a 6-yearold boy. There was consanguinity in the family, both parents were healthy. The birth weight, early mental and motor developmental milestones were normal for both siblings. Difficulty in walking appeared at the age of five in patient 1 and at the age of four in patient 2. Subsequently, joint pain, joint swelling, stiffness and enlargement of the proximal interphalangeal joints (Figure 1a) developed over time. The stature of the patients was in the normal range. The rheumatological serology and routine biochemical tests were normal. The irregular acetabular roof, arthrotic changes of the femoral heads, anterior ossification defects in vertebral

Address for Correspondence

University of Health Sciences, Ankara Child the alth and Diseases Hematology Oncology Training and Research Hospital, Clinic of Pediatric Genetics, Ankara, Turkey Phone: +90 5325539876 E-mail: korkmazkilic@yahoo.com.tr ORCID ID: orcid.org/0000-0003-0522-1809 Received: 17.11.2016 Accepted: 11.01.2017

${ }^{(\odot)}$ Copyright 2018 by Ege University Faculty of Medicine, Department of Pediatrics and Ege Children's Foundation The Journal of Pediatric Research, published by Galenos Publishing House. 


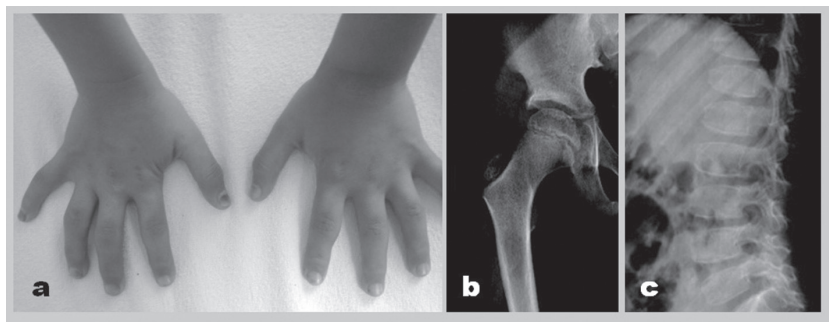

Figure 1. a) Clinical photographs and $X$-ray films of patient 1 show an enlargement of the proximal interphalangeal joints, b) irregular acetabular roof, c) flattening and anterior beaking on the thoraco-lumbal spine

bodies and expanded articular ends of the tubular bones with mild narrowing joint space were seen in X-rays (Figure 1b, c). Diagnosis was made combining clinical and radiographic findings. The genomic DNA of both siblings and parents were screened for mutations using a Sanger sequencing. The mutational analysis of WISP3 revealed a known disease causing homozygous c.793-794 delTG (p.C265Lfs*31) mutation in both siblings. The parents have the same heterozygous state WISP3 mutation.

\section{Discussion}

PPRC can be defined as non-inflammatory chondropathy affecting mainly articular cartilage. Any depletion or dysfunction of WISP3 can result in cartilage lesion (5). The relationship between phenotype and genotype of PPRC is still unclear. The characteristic clinical findings are as follows; platyspondyly and progressive arthropathy resulting in short stature, pain, stiffness and swelling of the joints without synovitis. The characteristic radiographic features are narrow joint space with wide metaphyses, flat epiphyses, enlarged femoral heads with irregular acetabular margins. In the patients presented in this study, the phenotypic, radiographic and serologic features of the patients were in accordance with the literature; both siblings had multiple joint deformities (stiffness and swelling), slightly undersized long bones, progressive loss of joint space, mild osteoporosis, widened epiphyses, vertebral flattening and anterior beaking and normal blood laboratory findings. Similar to the literature, the age of onset was 5 years in the patients presented in this study. In PPRC, joint stiffness usually first affects the hips and progressively involves the other joints, especially the proximal and distal interphalangeal joints. In the patients presented in this study, both siblings had stiffness and osteoarthrosis on both hip joints and an enlargement of interphalangeal joints. Growth is retarded in PPRC, with a final height between 135 and $150 \mathrm{~cm}$ but these siblings did not exhibit short stature at the time of the study. PPRC is often misdiagnosed as IIA. The differential diagnosis includes rheumatic diseases of childhood but in PPRC, there is an absence of soft tissue involvement. In PPRC, there is no synovitis or joint inflammation. The other conditions that may be considered in the differential diagnosis are spondyloepiphyseal dysplasia, spondylo-metaphyseal condrodysplasia and lysosomal storage disorders. Unlike other congenital skeletal disorders, patients with PPRC are often asymptomatic in the first years of life. Although life expectancy is normal, the prognosis of PPRC can be very poor. As a result of progressive artropathy, most patients are wheelchair-bound from an early age (5). The appropriate differential diagnosis and early recognition of PPRC is critical for treatment management and genetic counselling because anti-inflammatory drugs are not efficient in PPRC (5). In conclusion, early recognition and genetic counselling are essential for families with PPRC in order to avoid unnecessary treatment and further recurrence. Pediatricians, rheumatologists, endocrinologist and orthopaedists should be familiar with the phenotypic and radiographic features of PPRC, especially when dealing with sero-negative arthritis in consanguineous families. Accurate diagnosis of PPRC is essential to avoid misdiagnosis and unnecessary treatment for IIA. Early recognition and genetic counselling is vital for families where there is risk of a birth of a second affected child before the first one is diagnosed.

\section{Ethics}

Informed Consent: Was obtained from parents.

Peer-review: External and internal peer reviewed.

Financial Disclosure: This study has received no financial support.

\section{References}

1. Spranger I, Albert C, Schilling F, Bartsocas C, Stöss H. Progressive pseudorheumatoid arthritis of childhood (PPAC). A hereditary disorder simulating rheumatoid arthritis. Eur I Pediatr 1983;140:34-40.

2. Hurvitz JR, Suwairi WM, Van Hul W, et al. Mutations in the CCN gene family member WISP3 cause progressive pseudorheumatoid dysplasia. Nat Genet 1999;23:94-8.

3. Nakamura $\mathrm{Y}$, Weidinger $\mathrm{G}$, Liang $\mathrm{J}$, et al. The CCN family member Wisp3, mutant in progressive pseudorheumatoid dysplasia, modulates BMP and Wnt signaling. I Clin Invest 2007;117:3075-86

4. Ehl S, Uhl M, Berner R, Bonafé L, Superti-Furga A, Kirchhoff A. Clinical, radiographic, and genetic diagnosis of progressive pseudorheumatoid condrodysplasia in a patient with severe polyarthropathy. Rheumatol Int 2004;24:53-6.

5. Garcia Segarra N, Mittaz L, Campos-Xavier AB, et al. The diagnostic challenge of progressive pseudorheumatoid dysplasia (PPRD): a review of clinical features, radiographic features, and WISP3 mutations in 63 affected individuals. Am J Med Genet C Semin Med Genet 2012;160:217-29. 